\title{
SIÈCLE D’AUGUSTE ET ÂGE D’OR
}

\begin{abstract}
Summary: We find three definitions of the saeculum in the Roman world. The Etruscan Century is based on the lives of the human beings and of the different cities in Etruria. We find an echo of these theories in the Roman divination There are two definitions of the century in Rome, a century of 100 and a century of 110 years. This theory, elaborated by the Quindecemviri sacris faciundis and Ateius Capito, has been taken into consideration to celebrate the Secular Games in 17 AD. In fact, the reign of Augustus has been considered as the return of the Golden Age in Italy and in the Roman world, with the end of the civil wars. In Vergil's poetry, we find a historical conception of the Hesiodic Golden Age. Announced in the fourth Eclogue, the Golden age is localized in the Latium (Georgica) under the power of Augustus (Aeneid).
\end{abstract}

Key words: Augustus, Secular Games (Ludi Saeculares), Golden Age, Vergil

D'un point de vue historique, la notion de «siècle » est attachée à un personnage pour définir une période de l'histoire plutôt glorieuse. Cette notion a été illustrée par Voltaire qui a écrit un ouvrage intitulé Le Siècle de Louis XIV, publié en 1751. Mais, on parle aussi de «Siècle des Lumières » pour le XVIII ${ }^{\mathrm{e}}$ siècle français, qui a abouti à la Révolution, ou de «Siècle d'or » pour le rayonnement de l'Espagne en Europe aux $\mathrm{XVI}^{\mathrm{e}}$ et $\mathrm{XVII}{ }^{\mathrm{e}}$ siècles.

Il est en tout cas fort rare qu'un personnage attache son nom à une période assez illustre pour recevoir cette dénomination. Pour l'Antiquité, a très tôt été reconnu le Siècle de Périclès pour l'époque qui a vu l'éclosion du génie grec et la naissance des arts, des sciences et des lettres, pour reprendre une expression consacrée. Auguste a eu cet honneur dans le monde romain et l'on célèbre fort justement le Siècle d'Auguste ${ }^{1}$.

Le 19 août 14, Auguste mourait à l'âge de 75 ans, 10 mois et 26 jours, après avoir détenu l'imperium pendant près de 57 ans. Dès la mort d'Auguste, un sénateur a proposé, comme le précise très clairement Suétone dans sa biographie, que la période

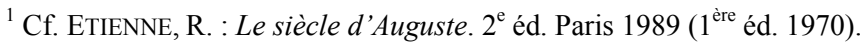


de l'histoire de Rome qui a été dominée par le Princeps reçût le nom de Siècle d'Auguste (ou « Siècle auguste », les deux notions se recouvrant en latin) et de l'inscrire dans les fastes, pour consacrer la gloire du fondateur de la nouvelle dynastie et de l'Empire. Il y avait là plus qu'une simple adulation de courtisan, mais presque une vision prophétique, car les historiens ont reconnu l'existence du Siècle d'Auguste.

Il est intéressant de mettre en parallèle, de confronter cette notion de «Siècle d'Auguste » avec celle de l'Âge d'or, puisque Virgile, lui-même prophète, vates plus que poeta, a célébré dès 41 dans la plus célèbre de ses Bucoliques, le retour de l'Âge d'Or à Rome : iam ... redeunt Saturnia regna ${ }^{2}$. Comment a pris forme cette notion de Siècle d'Auguste ? Sans doute, le rayonnement de la littérature, due en partie à Mécène $^{3}$, qui laissera d'ailleurs son nom au mécénat, y est-il pour quelque chose, l'âge d'or de la poésie latine coïncide avec le règne d'Auguste, mais ce n'est pas la seule raison. Plusieurs éléments entrent en ligne de compte et Auguste lui-même y fut pour quelque chose.

Qu'est-ce-ce qu'un siècle? Le substantif saeculum ne manque de poser problème en latin et n'a pas reçu d'étymologie sûre. Il est vrai que beaucoup de mots à diphtongue -ae- n'ont pas d'étymologie assurée. Plusieurs solutions ont pu être proposées, avec une fortune diverse.

Les rapprochements avec lat. senex, proposé par Varron ${ }^{4}$, et avec lat. sequor, présent dans les Étymologies d'Isidore ${ }^{5}$, sont sans aucune valeur, même s'ils insistent fort justement sur la durée de la vie humaine ; seul le celtique offre un rapprochement, où le gallois hoedl < saitlo- signifie précisément « durée de la vie », rapprochement peu significatif, dans la mesure où il est limité à deux langues ${ }^{6}$. Walde rattache à sero / seui / satum ce dérivé nominal en clo-, dont G. Serbat a souligné le vocalisme original et l'archaïsme ${ }^{7}$. À travers cette étymologie, un rapprochement a été suggéré avec le nom de Saturne, lié à l'Âge d'or, mais la quantité du -a- s'y oppose (on lit en effet sur une coupe l'inscription Saeturni pocolom ${ }^{8}$ ).

Si l'étymologie n'est pas établie, le sens du mot est clair, c'est celui de "génération, race, espèce ». En ce sens, on le trouve attesté chez Lucrèce, avec 42 emplois

\footnotetext{
${ }^{2}$ Verg. buc. IV 6.

${ }^{3}$ Cf. ANDRÉ, J.-M. : Mécène. Essai de biographie intellectuelle [Annales de l'Université de Besançon, vol. 86]. Paris 1967; ANDRÉ, J.-M. : Le siècle d'Auguste. Paris 1974.

${ }^{4}$ Varro, ling. 6. 11: seclum spatium annorum centum uocarunt, dictum a sene, quod longissimum spatium senecendorum hominum id putarunt.

${ }^{5}$ Isid. Orig. 5. 38. 1 : saecla generationibus constant, et inde saecula quod se sequantur, abeuntibus enim alii succedunt.

${ }^{6}$ ERNOUt, A. - MeILlET, A. : Dictionnaire étymologique de la langue latine, s. u. saeculum.

${ }^{7}$ LEW 2268; Serbat, G. : Les dérivés nominaux latins à suffixe médiatif. Lille 1976, 152. Cf. Nadjo, L. : Saeculum et la notion de fin de siècle. In Fins de siècle. Colloque de Tours, 5-6 juin 1985. Textes recueillis par P. CITTI. Bordeaux 1990, 37-48.

${ }^{8}$ CIL I I $^{2}$ 449, XI, 6708, 9; ERNOUT, A. : Textes latins archaïques. Paris 1957, nº108.
} 
au pluriel ${ }^{9}$, contre 4 au singulier ${ }^{10}$. De ce sens, on passe à celui de "durée d'une génération », sens attesté tout au long de la latinité, puis à celui de « longue période indéterminée $»$, sens déjà présent chez Virgile ${ }^{11}$, mais qui se généralisera surtout avec les auteurs chrétiens. Dans la Vulgate, saeculum traduit le grec aiôn des Septante ${ }^{12}$.

Le grec aiôn et le latin aetas dérivent d'une même racine indo-européenne qui désigne le temps considéré dans sa durée, par opposition à tempus, qui s'applique, tout au moins à l'origine, à un aspect ponctuel de la durée. Avec la forme aevus ou aevum, de la même famille (la forme aeuitas est attestée dans les XII Tables), la latin a conservé, sous forme d'un dérivé en -o-, le nom indo-européen de la « durée », de la « longue durée $»^{13}$.

Les deux notions se complètent et se rencontrent pour désigner le règne d'Auguste. On ne saurait parler de regnum, comme pour la période royale.

Le siècle est donc une longue durée, en rapport avec la vie humaine. Il n'en existe pas une définition unique, car les conceptions sont variables. À Rome, où les données latines ont été contaminées par des éléments étrusques, on va se trouver en présence de deux et même de trois définitions du siècle. À Rome, comme le précise Censorinus dans son opuscule Sur le jour natal, il existe un siècle naturel, fondé sur la plus longue durée de la vie humaine et un siècle civil ${ }^{14}$, dans le cadre de la cité, siècle auquel les autorités civiles et religieuses pourront attribuer une durée variable.

Le siècle d'Auguste se confond donc avec la vie d'Auguste, une vie marquée, dès sa naissance, par des prodiges qui annoncent un destin extraordinaire ${ }^{15}$; sa véritable naissance se situe toutefois plutôt en 44 av. J.-C., lorsqu'il entre en jeu sur la scène politique, comme l'héritier du dictateur assassiné ; la célébration des Jeux Séculaires sera le point culminant de cette vie et marquera le renouveau des temps dans le cycle de la Roma Aeterna.

L'œuvre d'Auguste s'inscrit dans la durée, dans le temps : Auguste met en application le calendrier julien de la réforme de 46 et laissera son nom au mois d'août. Avec Auguste, la durée de la vie humaine s'inscrit dans les siècles de Rome. La vie

${ }^{9}$ Lucr. $1.467 ; 1.339$ (saecula hominum) ; 2. $78 ; 5.855$ (animantum saecla) ; 2. $995 ; 2.1076$; $3.753 ; 4.413 ; 4.686 ; 5.1069 ; 6.1220$ (saecla ferarum)...

${ }^{10}$ Lucr. 2. $1079 ; 2.1169 ; 4.1227 ; 5.1521$.

${ }^{11}$ Verg. Aen. 6. 235 (per saecula).

${ }^{12}$ Cf. les expressions a saeculo (Ps. 92. 2 « de toute éternité »); in saeculum (Judith 13. 21); in saeculum saeculi (Ps. 9. 6 ; 18. 10); in saecula saeculorum (Eugippius, Vita sancti Seuerini 43. 7).

${ }^{13}$ ERNOUT-MEILLET, $D E$, s. u. aeuus/aeuum.

${ }^{14}$ Censorin. 17. 1 : et quoniam saecula aut naturalia aut ciuilia sunt, prius de naturalibus dicam. Saeculum est spatium uitae humanae longissimum partu et morte definitum.

${ }^{15}$ Suet. Aug. 94 : lors de la délibération au Sénat sur la conjuration de Catilina, le père d'Octave arriva en retard à cause de l'accouchement de sa femme et Nigidius Figulus, consultant les astres, assura qu'il était né un maître pour l'univers. Sur les biographies, cf. Nicolas de Damas, Vita Caesaris. In FHG (ed. Müller) Paris 1899, 99 sqq; FGH (ed. Jacoby) F. n 90, 125-130; HALL, C. M. : Nicolaus of Damascus, Life of Augustus. John Hopkins 1923 ; TURTURRO, G. : Vita di Augusto. Bari 1945. 
d'Auguste s'inscrit dans le cadre de la Grande Année (Annus Magnus), dans des cycles qui sont ceux de la Roma Aeterna.

La liste des fastes consulaires et triomphaux ${ }^{16}$ est affichée sur l'Arc d'Auguste au Forum (et non sur les parois de la Regia, comme on le lit quelquefois encore ${ }^{17}$ ); deux arcs ont été érigés par Auguste, le premier après la victoire d'Actium, en 29, le second après la restitution en 19 des enseignes prises à Crassus par les Parthes, en 53, et c'est sur l'arc parthique qu'il convient de placer les fastes consulaires et triomphaux. Les fêtes dynastiques vont prendre place dans les calendriers officiels. Alors que les événements de l'histoire annalistique étaient datés selon les fastes consulaires ou les grands événements comme les guerres puniques ou la prise de Rome par les Gaulois, le principat d'Auguste va désormais apparaître comme une référence.

Le Siècle d'Auguste est dominé par la célébration des Jeux Séculaires ${ }^{18}$. À la fin de la République et au début du principat, deux définitions du siècle sont en présence à Rome : un siècle de 100 et un siècle de 110 ans. Le problème se pose avec la célébration des Jeux Séculaires voulue sous le principat d'Auguste.

Varron, Valérius Antias et Tite-Live donnent au siècle une durée de 100 ans. TiteLive suit la chronologie établie par Valérius Antias selon laquelle les Jeux auraient été célébrés en $509^{19}, 449^{20}, 249^{21}$ et $149^{22}$ av. J.-C. Telle est la tradition annalistique.

Cependant, il existait une seconde tradition et Auguste a suivi une autre chronologie, s'appuyant sur une tradition apollinienne et sibylline, établie par le jurisconsulte Atéius Capito ${ }^{23}$, fondée sur un siècle de 110 ans, comme le souligne Horace dans son Carmen saeculare ${ }^{24}$; selon cette théorie, les jeux auraient été célébrés en 456, 346, 236 et 126 av. J.-C. Les Jeux auraient dû avoir lieu en 16 mais Auguste les associa au renouvellement de ses pouvoirs en 17 et ainsi les Jeux Séculaires furent des Jeux augustéens liés au pouvoir et à la personnalité du prince.

Il existe enfin une troisième théorie, celle des siècles étrusques, et sur ce point on va trouver encore une interférence avec la vie et les pouvoirs d'Auguste, en particulier pour la date de 44 av. J.-C.

${ }^{16}$ DEGRASSI, A. : Inscriptiones Italiae XIII 1: Fasti consulares et triumphales. Roma 1947 ; DEGRASSI, A. : L'edificio dei Fasti Consolari. RPAA 21 (1945-1946) 57-104.

${ }^{17}$ Tite-Live, Histoire romaine. Livre IV. Éd. J. BAYET. Paris 1954, 118.

${ }^{18}$ Cf. Pighi, J. B. : De ludis saecularibus populi Romani Quiritium libri sex. $2^{\mathrm{e}}$ éd. Amsterdam 1965.

${ }^{19}$ Censorin. 17. 10 : primos enim ludos saeculares exactis regibus post Romam conditam anno CCXLV a Valerio Publicola institutos esse Antias auctor est P. Valerio Sp. Lucretio consulibus.

${ }^{20}$ Censorin. 17. 10 : secundos ludos, ut Antias uult, anno post urbem conditam quinto trecentesimo. Le texte est corrompu et l'établissement difficile. Texte établi d'après Erycius Puteanus ; cf. Censorinus, De die natali liber. Ed. N. SALLMAN. Leipzig 1983, 36.

${ }^{21}$ Cenorin. 17. 10 : tertii ludi fecerunt Antiate Liuioque auctoribus P. Claudio Pulchro L. Junio Pullo consulibus (Liu. XIX $=$ frg. $9 \mathrm{Jal}$ ).

${ }^{22}$ Censorin. 1711 : de quartorum ludorum anno triplex opinio est. Antias enim et Varro et Livius relatos esse prodiderunt L. Marcio Censorino M. Manilio consulibus post Romam conditam anno sescentesimo quinto (Liu. XLIX = frg. $14 \mathrm{Jal})$.

${ }^{23}$ Zosimus 2. 4. 2.

${ }^{24}$ Hor. carm. saec. 21-24. 
Le principat d'Auguste n'est pas étranger à la théorie étrusque des siècles, consignée dans les Livres des Destins, libri fatales des Étrusques, livres qui faisaient eux-mêmes partie des Libri rituales ${ }^{25}$ : on y décèle un courant hostile au principat, bien perceptible dans la tradition. Dans ce cadre étrusco-latin, c'est l'année 44 qui est au centre des spéculations. Les siècles étrusques sont des siècles naturels, liés, pour chacune des cités de la dodécapole, à la plus vie des individus : le premier siècle est défini par celui des individus nés le jour de la fondation qui atteint la plus grande longévité ; la durée du second siècle est déterminée par la durée maximale de la vie d'un individu né le jour de l'expiration du premier siècle. Les siècles s'enchaînent ainsi selon des durées variables. Censorinus, qui s'appuie sur le témoignage de Varron et des Tuscae Historiae écrites au cours du VIII ${ }^{\mathrm{e}}$ siècle, pour nous préciser la durée des siècles de la nation étrusque, donne les précisions suivantes : les quatre premiers siècles ont eu une durée de cent ans, le cinquième a compté 123 ans, le sixième et le septième 119 ans $^{26}$.

Ce huitième siècle est un repère particulièrement important : il se retrouve mentionné, en liaison avec l'inuidia hominum, dans un texte fondamental, la Prophétie dite de Végioa ${ }^{27}$, un document exceptionnel qui nous a été conservé dans un traité tardif d'arpentage ${ }^{28}$.

Les historiens s'appuient sur un passage de la Vie de Sylla de Plutarque ${ }^{29}$, sur une notice de Servius ${ }^{30}$ et sur un passage de Dion Cassius pour préciser les limites des VIII $^{\mathrm{e}}$ et IX ${ }^{\mathrm{e}}$ siècles étrusques. Les prodiges de l'année 88 peuvent être mis en rapport avec le début du $\mathrm{IX}^{\mathrm{e}}$ siècle et ce $\mathrm{IX}^{\mathrm{e}}$ siècle étrusque aurait pris fin en 44 , année marquée par l'apparition d'une comète signalée lors de la célébration des Jeux Funèbres célébrés par Octave en l'honneur de César. Un commentaire de Servius Danielis ${ }^{31}$

${ }^{25}$ Cf. Thulin, C. : Die etruskische Disciplin. T. III: Die Ritualbücher [Göteborgs Högskolas Arsskrift, Band XV] Göteborg 1909. Réédition: THulin, C. O. : Die Etruskische Discipline. Teil I-III. Darmstadt 1968.

${ }^{26}$ Censorin. 17. 6 : haec portenta Etrusci pro haruspicii disciplinaeque suae peritia diligenter obseruata in libros rettulerunt. Quare in Tuscis Historiis, quae octauo eorum saecula scriptae sunt, ut Varro testatur, et quot numero saecula ei genti data sint et transactorum singula quanta fuerint quibusue ostentis eorum exitus designati sint continetur. Itaque scriptum est quattuor prima saecula annorum fuisse centenum, quintum centum viginti trium, sextum undeuiginti et centum, septium totidem, octauum tum demum agi, nonum et decimum superesse, quibus tansacti fine fore nominis Etrusci.

${ }^{27}$ Heurgon, J. : The date of Vegoia's prophecy. JRS 49 (1959) 41-51 ; TURCAN, R. : Encore la prophétie de Végoia. In L'Italie préromaine et la Rome républicaine. Mélanges offerts à Jacques Heurgon. Vol. II. Rome 1976, 1009-1019; ZANCAN, L. : Il frammento di Vegoia e il nouissimum saeculum. Atene e Roma III 7 (1939) 203-219 ; MAZZARINO, S. : Il pensiero storico classico. Paris 1966, II 525 ; VALVO, A. : La profezia di Vegoia. Rome 1988.

${ }^{28}$ Après avoir été publié par A. Turnèbe en 1554 (editio princeps ) et par Willem van Goes (Goesius) en 1674, le corpus des Gromatici Veteres a connu une mise au point scientifique à travers l'ouvrage de référence Blume, F. - LACHMANN, K. - RudorfF, A. : Schriften der Römischer Feldmesser. Bd. I. Berlin 1848, 350-351 ; cf. REEVE, M. D. : Agrimensores. In REYNOLDS, L. D. (ed.) : Texts and Transmission. A Survey of the Latin Classics. Oxford 1983, 16 ; DILKE, O. A. W. : The Roman Land Surveyor. An Introduction to the Agrimensore. Newton Abbot 1971, 126-132, et 227-230.

${ }^{29}$ Plut. Sylla 7. 6-11.

${ }^{30}$ Serv. Dan. ad Verg. Aen. VIII 526.

${ }^{31}$ Serv. Dan. ad Buc. IX 46 (cf. Serv. ad Aen. I 287) ; Obs. 68 (ex Liui libro CXVII) ; Plin. H. N. II 93-94 ; Sen. Q. N. VII 17. 2 ; Suet. Caes. 88 ; Plut. Caes. 69 ; Cass. Dio XLV 7.1 ; Lyd. ost. 10 B. 
nous a préservé l'interprétation étrusque du phénomène donnée par un haruspice appelé Vulcatius (ou Vulcanius), qu'il a trouvé chez l'historien Baebius Macer et dans le De Vita sua d'Auguste : le prodige signalait la fin du $\mathrm{IX}^{\mathrm{e}}$ siècle et le début du $\mathrm{X}^{\mathrm{e}}$ siècle. Après la révélation de ce secret, l'haruspice Vulcanius s'écroula mort en pleine assemblée. Cette version est en tout cas celle de la propagande augustéenne mais on trouve une version moins favorable chez Appien, une version plus étrusquisante, selon laquelle cette mort serait une mort volontaire, justifiée par la volonté d'échapper à la tyrannie ${ }^{32}$. L'entrée en scène d'Octave coïncide avec le début du $\mathrm{X}^{\mathrm{e}}$ siècle étrusque.

Aux événements des années 88 et 44 av. J.-C., on peut joindre, comme le font W. V. Harris et D. Briquel, des événements survenus en 19 ap. J.-C. et signalés par Dion Cassius : sonnerie de trompette chez le consul Norbanus le premier jour de l'année, chute d'une statue de Janus, oracle sibyllin liant le destin de Rome à un cycle de 900 ans $^{33}$. Ainsi se trouverait accompli le cycle des siècles dits étrusques.

Non seulement le règne d'Auguste est en lui-même le Siècle d'Auguste, mais il est identifié aussi comme un nouvel âge d'or, comme marquant le retour de l'âge d'or dans le monde romain. Comment expliquer ce rapprochement, qui n'a rien de fortuit et qui repose sur une subtile et savante propagande ?

À Rome, au premier siècle avant notre ère, le thème de l'Âge d'Or n'est pas nouveau; déjà, Catulle l'a évoqué dans son grand poème ${ }^{34}$, mais tous les poètes du siècle d'Auguste, Horace ${ }^{35}$, Ovide $^{36}$ et surtout Virgile ${ }^{37}$ le mettent à l'honneur. C'est Virgile qui va conférer au thème sa véritable dimension en l'inscrivant dans l'histoire,

${ }^{32}$ Appian. B C IV 4.

${ }^{33}$ Dio Cass. 57. 18. 3-5 ; HARRIS, W. V. : Rome in Etruria and Umbria. Oxford 1971, 35 ; BRIQUEL, D. : Les changements de siècles en Etrurie. In Fins de siècle (n. 7) 65-66.

${ }^{34}$ Catull. LXIV 384-393.

${ }^{35}$ Hor. ep. XVI 41-66. Cf. GRIMAL, P. : À propos de la XVI épode d'Horace. Latomus XX (1961) 721-730; MAC LEOD, C. W. : Horace and the Sibyl (Epode XVI, 2). CQ XXXIX (1979) 220-221.

${ }^{36}$ Ovid. met. I 89-112; am. III 8. 35-44 ; fast. I 193.

${ }^{37}$ Parmi une bibliographie très riche, cf. surtout, dans le cadre restreint de cette étude : KUBUSCH, K. : Aurea Saecula: Mythos und Geschichte. Untersuchung eines Motivs in der antiken Literatur bis Ovid. Frankfurt 1986 ; BRISSON, J. P. : Rome et l'âge d'or, de Catulle à Ovide, vie et mort d'un mythe. Paris 1992 ; BRISSON, J. P. : Rome et l'âge d'or : fable ou idéologie? In Poikilia. Études offertes à J. P. Vernant. Paris 1987, 123-143 ; BRISSON, J. P. : Rome et l'âge d'or: Dionysos ou Saturne ? MEFRA 100.2 (1988) 917-982 ; BRISSON, J. P. : Jupiter, Dionysos et l'âge d'or aux derniers temps de la République romaine, un débat de société. In L'âge d'or. Sous la direction de J. POIRIER. Dijon 1996, 63-73; GUITTARD, CH. : Recherches sur la nature de saturne, des origines à la réforme de 217 av. J.-C. In BLOCH, R. (dir.) : Recherches sur les religions de l'Italie antique. Genève 1976, 43-71 ; BRISSON, J. P. : « Saturnia terra », mythe et réalité. In Actes du Colloque Histoire et historiographie. 8-9 décembre 1978 [Caesarodunum XV bis]. Paris 1980, 177-186. En ce qui concerne le dieu lui-même, trois monographies demeurent fondamentales : SiPPEL, G. : De cultu Saturni. Marburg 1848 ; ALBRECHT, J. : Saturnus, seine Gestalt im Sage und Kultus. Halle 1943 ; LE GLAY, M. : Saturne africain. Histoire [Bibl. des Écoles françaises d'Athènes et de Rome $n^{\circ}$ 205] Paris, 1966, 449-478. Cf. aussi PAVAN, M. : Aurea (aetas ; gens ; saecula). In Enciclopedia Virgiliana. Roma, I (1984) 412-416. 
précisément dans le siècle d'Auguste, et dans un espace défini qui n'est autre que l'Italie. Après avoir annoncé le retour des Saturnia regna dans la célèbre quatrième églogue, cette conception va se préciser dans les Géorgiques et une évolution, même une rupture, est notable ${ }^{38}$.

Virgile abandonne le thème d'un retour de l'Âge d'or, renonçant à l'optique mystique, millénariste et eschatologique qui était celle des néo-pythagoriciens : ce revirement peut s'expliquer par la déception qui a suivi la paix de Brindes, l'éloignement de Pollion de la vie politique, éléments qui ne sont sans doute pas étrangers à ce renoncement. Le poète va évoluer vers une vision plus raisonnée, plus personnelle et cohérente de l'Âge d'or. Il passe à une optique historique, géographique et morale, qui sera définitivement la sienne : c'est l'Âge d'or de la Saturnia tellus, que Saturne avait fait régner jadis dans un pays particulier, à savoir l'Italie.

Après s'être démarqué des conceptions hésiodiques dans le chant I des Géorgiques $^{39}$, en faisant du mythe le véhicule de l'idée d'un progrès de l'humanité, le poète développe à loisir une conception personnelle dans le chant II, en deux étapes, l'idéalisation de l'Italie ${ }^{40}$ et l'éloge de la vie des paysans ${ }^{41}$. L'Italie apparaît alors comme la terre par excellence de l'Âge d'or et les paysans italiens comme les héritiers de la race d'or.

L'exaltation de l'Italie devient à la fin de la République un élément important de la nouvelle idéologie qui se mettra en place avec Auguste. On en retrouve des éléments chez Varron, dans le livre I des Res rusticae ${ }^{42}$, et Denys d'Halicarnasse ${ }^{43}$ : la laus Italiae met en valeur une opposition entre une Italie gouvernée et protégée par Octave et l'Orient dominé par Antoine et, plus largement, entre l'Europe et l'Asie. Ce thème justifie la domination de Rome et de l'Italie sur les provinces et vient appuyer un éloge de la vie rurale associant le labor humain à la générosité de la nature. Peu à peu va se dégager une vision unitaire de la péninsule telle qu'elle s'impose à l'époque d'Auguste.

Avec l'éloge de l'Italie, le cadre de l'Âge d'or virgilien est posé : son espace est la Saturnia tellus, son temps, le présent immédiat. Il restait au poète à définir avec plus de précision la composante humaine de sa vision, en précisant qui sont les héri-

${ }^{38}$ Cf., sur ce point, notre étude, « Aurea aetas » et « Saturnia tellus » dans la poésie virgilienne, dans Clacissismus und Modernität (Szeged, 11-13 septembre 2003). Acta Antiqua Archeologica, Szeged (Hongrie) XXX (2007) 71-90.

${ }^{39}$ Verg. georg. I 121-146.

${ }^{40}$ Verg. georg. II 136-176.

${ }^{41}$ Verg. georg. II 458-540.

${ }^{42}$ Varro, res rust. I 2. 3-7. Cf. le commentaire de J. HEURGON : Varron. L'économie rurale. I : Livre I. Paris 1978, 105-106. Pour R. REITZENSTEIN (Die geographischen Bücher des Varro. Hermes 20 [1886] 514-551), ce passage se serait également trouvé dans le livre XI des Antiquitates. Pour R. MARTIN (Recherches sur les agronomes latins et leurs conceptions économiques et sociales. Paris 1971, 275-286), un tel éloge de l'Italie implique une rédaction ancienne, autour de 57 av. J. C. et ne saurait correspondre aux périodes plus récentes qui ont vu des remaniements de l'ouvrage ; après les ravages causés par les guerres civiles en Italie, un tel éloge paraît déplacé. Mais un tel topos de la littérature peut être indépendant des réalités politiques et économiques.

${ }^{43}$ Dionys. Hal. I 36-37. 
tiers de la race d'or, parmi les Italiens : c'est ce qu'il fait à la fin du chant II, en dépeignant la perfection de la vie des paysans.

L'idée d'un règne de Saturne établi parmi les hommes en Italie trouvera son expression la plus achevée dans l'Énéide, au chant VIII, lors de la promenade d'Évandre et d'Enée sur le site de la future Rome ${ }^{44}$. L'idée d'un Âge d'or lié au règne de Saturne, sur le site de Rome, trouve son expression poétique la plus accomplie chez Virgile. Ne peut-on aller jusqu'à dire qu'elle est une invention du poète, favorisée par les conceptions ambiantes, les considérations sur une géographie mythique du Latium, l'élaboration des royautés primitives?

Lors de leur promenade au pied du Capitole, Évandre évoque devant Énée le règne du dieu civilisateur et législateur sur le Latium, où Saturne aurait trouvé refuge et se serait caché, après avoir été détrôné par Jupiter. C'est alors que Virgile fait état de cette curieuse étymologie du nom du Latium, qui tirerait son nom de ce qu'il aurait offert une cachette au dieu détrôné par les Olympiens ${ }^{45}$.

Avant Virgile, les sources grecques demeurent muettes, en tout cas ne sont guère explicites sur un éventuel règne de Saturne sur le Latium : Diodore évoque bien un règne de Saturne in terris au livre $\mathrm{V}$ de sa Bibliothèque historique ${ }^{46}$, mais ce règne s'étend sur toute l'Hespérie et se déroule avant l'accession au pouvoir de Jupiter. Les sources latines sur ce sujet se réduisent à deux auteurs : Ennius et Varron.

Comme Virgile, Varron mentionne le nom de Saturne à propos de la toponymie du Capitole $^{47}$; l'Italie elle-même aurait été dénommée terre de Saturne ${ }^{48}$; un oppidum du nom de Saturnia aurait occupé la colline. Selon Servius ${ }^{49}$, 1'Italie aurait été successivement appelée Hesperia, Ausonia, Saturnia, et seulement enfin Italia $^{50}$. Selon Antiochos de Syracuse, le terme d'Italie, comme celui d'Oenotrie, désigna d'abord

${ }^{44}$ Verg. Aen. VIII 306-358.

${ }^{45}$ Verg. Aen. VIII 319-325. Cf. Seru. ad Aen. VIII 329 : bene «maluit» : nam et Saturnia dicta est, ut et nomen posuit Saturnia tellus. Et Vergilius Latium uult dici quod illic Saturnus latuit (fugiens Iouem)... Varro autem Latium dici putat quod latet inter praecipitia Alpium et Apennini.

${ }^{46}$ Diod. V 66.

${ }^{47}$ Varro, ling. V 42 : hunc antea montem Saturnium appellatum prodiderunt et ab eo late Saturniam terram ut etiam Ennius appellat. Antiquum oppidum in hoc fuisse Saturnia scribitur. Eius uestigia etiam nunc manent tria, quod Saturni fanum in faucibus, quod Saturnia porta, quam Iunius scribit ibi, quam nunc uocant Pandanam, quod post aedem Saturni in aedificiorum legibus priuatis parietes postici muri sunt scripti. Cf. De lingua Latina. Livre V. Éd. du J. COLlarT. Paris 1954, $26-27$ et 169-170 ; POUCET, J. : Recherches sur la légende sabine des origines de Rome. Louvain 1967, 76-96 ; GUITTARD, CH. : « Saturni fanum in faucibus » (Varro, L. L. 5. 42) : à propos de Saturne et de l'Asylum. In LE BONNIEC, H. - VALLET, G. (éd.) : Mélanges de littérature et d'épigraphie latines, d'histoire ancienne et d'archéologie. Hommage à la mémoire de Pierre Wuilleumier. Paris 1980, 159-166 ; GuITTARD, CH. : La topographie du temple de Saturne d'après la notice varronienne du De lingua Latina (V, 42). In CHEVALLIER, R. (éd.) : Présence de l'architecture et de l'urbanisme romains. Hommage à Paul Dufournet. Paris 1983, 31-39.

${ }^{48}$ Sur cette tradition, cf. aussi Dionys. Hal. I 34. 4 et VI 1. 4 ; Tert., apol. X 8 et ad nat. II 12 ; Fest. 430L ; Iust. XLIII 1. 5 ; Solin. I 12 ; Vib. Seq. geogr. p. 157 Riese ; Macr. sat. I 7. 27.

${ }^{49}$ Seru. ad Aen. VIII 322 : at Italia plura nomina habuit, dicta est enim Hesperia, Ausonia, Saturnia, Italia.

${ }^{50}$ GÉLY, S. : Le nom de l'Italie : Mythe et Histore d'Hellanicos à Virgile. Genève 1992, 339-351. 
l'extrême pointe du Bruttium, au sud de l'isthme compris entre le golfe de Térina et le golfe de Scyllétion ${ }^{51}$.

Varron ne mentionne jamais un règne de Saturne sur le Latium. Il évoque, d'un point de vue toponymique, l'extension du concept de Saturnia à l'ensemble de l'Italie : encore son allusion demeure-t-elle dépourvue de limites géographiques précises (et ab eo late Saturniam terram $)^{52}$. Il corrige ainsi dans un sens romano-centrique l'histoire du concept d'Italie qui s'est d'abord appliqué à la pointe du Bruttium. Plusieurs éléments ont joué dans cette construction. La présence du temple de Saturne, consacré en 497 av. J.-C. au pied du Capitole ${ }^{53}$, abritant l'aerarium, le trésor public du peuple romain $^{54}$, la célébration des Saturnales, grande fête de décembre abolissant les distinctions sociales.

Selon Macrobe, les récits étiologiques sur l'institution des fêtes en l'honneur de Saturne sont au nombre de trois et tous soulignent le rôle civilisateur de Saturne ; le premier est en rapport avec la royauté de Janus sur l'Italie et le partage du pouvoir dans une dyarchie harmonieuse ${ }^{55}$; le second fait état des migrations pélasgiques, en relation avec l'oracle de Dodone ${ }^{56}$; enfin, un récit est plus particulièrement en rapport avec le séjour d'Hercule en Italie et l'épisode des bœufs de Géryon ${ }^{57}$. L'idée d'une royauté partagée entre Janus et Saturne est rapportée à Caius Julius Hyginus, préfet de la bibliothèque palatine, ami d'Ovide et polygraphe. Hygin cite Protrarchos de Thralles.

Lactance nous livre, au livre I de ses Institutions divines, d'après l'Histoire sacrée d'Ennius, une version détaillée et complexe de la rivalité entre Saturne et Jupiter : Jupiter libère Saturne emprisonné par Titan ; mais Saturne, mis en garde par un oracle, veut éliminer, en lui tendant un piège, Jupiter qui parvient à le renverser et à le chasser ;

${ }^{51}$ Dionys. Hal. I 12 ; I 35 ; I 73.

${ }^{52}$ Cf. COLlaRT (n. 47) 26-27, 127, 169-170) qui traduit : « Selon la tradition, cette colline s'appelait auparavant Mons Saturnius (Mont de Saturne) et tout le continent environnant Saturnia terra (terre de Saturne). » L'emploi de l'adverbe late peut ne concerner que le pays environnant, à savoir le Latium ; on retrouverait même entre l'adverbe late et le nom Latium un jeu étymologique, sans fondement, au mépris des quantités. Cf. la traduction de P. MONAT : «Il leur donna des lois, voulut que le pays / fût appelé Latium, car toute latitude / de se cacher en paix il y avait trouvé. » (Lactant. inst. diu. I 13. 9. Éd. Sources chrétiennes $\mathrm{n}^{\circ}$ 326. Paris 1986, 145).

${ }^{53}$ Liu. II 21. 1; cf. Macr. sat. I 8. 1; Dion. Hal. VI 1. 4. E. GJERSTAD (The Temple of Saturn in Rome, Its Date of Dedication and the early History of the Sanctuary. In Hommages à Albert Grenier. Latomus LVIII 2 [1962] 757-762) restitue la chronologie suivante : fanum primitif comprenant une simple ara (époque de Tullus Hostilius); début des travaux de construction par Tarquin l'Ancien; dédicace par le dictateur Titus Larcius en 497 av. J.-C. Cf. LugLi, G. : Roma antica, il centro monumentale. Roma 1946, 148-151; COARELLI, F. : Il comizio dalle origini alla fine della repubblica, cronologia e topografia. PP XXXII (1977) 166-288; COARELli, F. : Il Foro Romano. Vol. I. Roma 1983, 199-226; COARELLI, F. : Guida archeologica di Roma. Verona 1974, 72-74; GUITTARD : Saturni fanum (n. 47)159-166.

${ }^{54}$ Sur cette fonction, cf. CORBIER, M. : L' « aerarium Saturni » et l' " aerarium militare ». Administration et prosopographie sénatoriale [Coll. EFR 24]. Roma 1974.

${ }_{55}^{55}$ Macr. sat. I 7. 19-26.

${ }^{56}$ Macr. sat. I 7. 28; Dionys. Hal. I 19. 3. Cf. BRIQuel, D. : Les Pélasges en Italie [Bibl. des Écoles françaises d'Athènes et de Rome 252]. Rome 1984, 355-406 ; GUITTARD, CH. : Recherches sur la nature de Saturne des origines à la réforme de 217 avant J.-C. In BLOCH, R. (éd.) : Recherches sur les réligions de l'Italie antique. Genève-Paris 1976, 58-60.

${ }^{57}$ Macr. sat. I 7. 27. 
Saturne parvient à se réfugier en Italie ${ }^{58}$. La dernière phrase est capitale : « Rejeté de pays en pays, poursuivi par des gens armés que Jupiter avait envoyés pour l'arrêter ou le tuer, Saturne trouva à grand peine en Italie un endroit où se cacher. » L'endroit où se cacher évoque le rapport latere / Latium, présent chez Virgile.

Cette référence à Ennius est le seul témoignage formel, dans les textes antérieurs à Virgile, qui évoque la fuite de Saturne dans le Latium et la volonté d'y trouver une cachette, ce qui implique un lien étymologique entre le toponyme et le verbe latere. Or, ce passage de l'Histoire sacrée ne nous est connu que par les Institutions divines de Lactance et aucune autre source ne vient le corroborer. Aussi est-on autorisé, avec M. Wifstrand-Schiebe ${ }^{59}$, mettre en doute le rattachement de cette donnée au texte d'Ennius. On remarque en effet que, si le début du passage, introduit par « reliqua Historia sic contextitur », est au style indirect, la dernière phrase est au style direct et semble marquer une rupture de construction, confirmée par le recours au relatif de liaison, qui est plutôt rare dans la langue d'Ennius et le latin archaïque et que Lactance utilise volontiers.

Si l'on tient compte des intentions de Varron et de l'importance du dieu Saturne dans sa problématique, on s'étonne qu'il ne soit jamais directement question de la royauté de Saturne sur le Latium et qu'il ne considère pas le dieu comme le fondateur de la mythique Saturnia. Si la légende du livre VIII de l'Énéide avait été établie à l'époque de Varron, il est curieux qu'il n'en soit pas question dans le De lingua Latina.

Que l'on trouve chez Virgile un écho des théories varroniennes ne fait pas de doute : Virgile ne pouvait ignorer Varron et cette notice est le reflet d'une réflexion sur les origines et la toponymie capitoline. Mais rien ne prouve que Varron ait connu et développé le thème du règne de Saturne sur le Latium. On ne trouve même pas ici mention de la fausse étymologie mettant le nom du Latium avec la cachette (latere) que le dieu y aurait trouvé au moment de sa disgrâce et de sa chute ${ }^{60}$; le lien avec l'adverbe late est assez lâche et plutôt allusif. Bien plus, dans un autre passage, Varron met le nom du Latium en relation étroite avec le nom du roi Latinus dont il n'hésite pas à le faire dériver ${ }^{61}$. Quant à Servius, il renvoie bien à Varron à propos de cette étymologie mais sans reprendre le thème de l'exil de Saturne et en avançant une

\footnotetext{
${ }^{58}$ Lactant. diu. inst. I 14. 10-12 : Reliqua Historia sic contextitur : Iouem adultum, cum audisset patrem atque matrem custodiis circumsaeptos atque in uincula coniectos, uenisse cum magna Cretensium mutitudine Titanumque ac filios eius pugna uicisse, parentes uinculis exemisse, patri regnum reddidisse atque ita in Cretam remeasse. Post haec deinde Saturno sortem datam ut caueret ne filius eum regno expelleret; illum eleuandae sortis atque effugiendi periculi gratia insidiatum Ioui, ut eum necaret; Iouem cognitis insidiis regnum sibi denuo uindicasse ac fugasse Saturnum. Qui cum iactatus esset per omnes terras persequentibus armatis, quos ad eum comprehendedum uel necandum Iuppiter miserat, uix in Italia locum in quo lateret inuenit. (1986) 43-60

${ }^{59}$ WifSTRAND-SCHIEBE, M. : The Saturn of the Aeneid, tradition or innovation? Vergilius 32

${ }^{60}$ Verg. Aen. VIII 322-323.

${ }^{61}$ Varro, ling. V 32: qua regnum fuit Latini, universus ager dictus Latius, particulatim oppidis cognominatus, ut a Praeneste Praenestinus, ab Aricia Aricinus. Cf. Collart (n. 47) 165, ad loc. : «Varron semble faire venir Latius (et Latium par conséquent) de Latinus. D’autres philologues anciens inversement, font venir Latinus de Latium. Toutefois, l'explication la plus fréquente qu'on trouve chez les grammairiens apparente Latium à lateo (se cacher). "
} 
explication pour le moins curieuse, sur la situation géographique de l'Italie : quod latet Italia inter praecipitia Alpium et Appennini. On trouve donc deux dérivations $L a$ tium $<$ Latinus et Latium $<$ latere, qui ne prennent pas en compte le mythe de Saturne.

Cette notion de « saeculum » liée au principat, inaugurée sous le règne d'Auguste, va désormais s'inscrire dans l'histoire de l'Empire et des Jeux Séculaires seront célébrés régulièrement, sans toutefois respecter un comput régulier : Claude et Antonin célébreront la $800^{\mathrm{e}}$ et la $900^{\mathrm{e}}$ année de Rome, Philippe le millenium, le 21 avril 248. Domitien célébrera des Jeux en 88 et Septime Sévère en 204 de notre ère. Claude n'a pas tenu compte de la célébration d'Auguste et a célébré ses jeux qui coïncidaient avec la $800^{\mathrm{e}}$ année de Rome. On voit que les principes ont tenu à faire coïncider la notion de Siècle avec leur propre règne. Ce lien entre le règne d'Auguste et le retour d'un Âge d'or avec la restauration de la paix civile est désormais bien établi, en particulier grâce au génie virgilien qui, dans toutes ses œuvres, des Bucoliques à l'Énéide, en a célébré la portée.

Charles Guittard

Université Paris-Ouest-Nanterre

charles.guittard@u-paris10.fr 